\title{
QUALIDADE PERCEBIDA PELO CLIENTE EM ATENDIMENTO EMERGENCIAL HOSPITALAR: Um ensaio teórico.
}

\section{QUALITY PERCEIVED BY THE CUSTOMER IN EMERGENCY HOSPITAL SERVICE: A theoretical test.}

\author{
Fernanda Pereira da Silva Carpeggiani ${ }^{1}$ \\ Ana Paula Affonso Gomes ${ }^{2}$ \\ Anna Maria Barancelli ${ }^{3}$ \\ Luiza Onzi Cavagnoli ${ }^{4}$ \\ Marcelo Luiz Batista ${ }^{5}$ \\ Mellina da Silva Terres 6
}

\begin{abstract}
Resumo
As emergências hospitalares são locais de prestação de serviços de grande importância social, possuindo alta complexidade e peculiaridade, portanto a prática da qualidade adquire enfoque e diferencial específicos. $\mathrm{O}$ presente trabalho tem como objetivo, propor o desenvolvimento de um modelo teórico de HEALTHQUAL aplicável ao contexto de serviços de emergência hospitalar, visando mensurar os indicadores de qualidade percebida durante o atendimento emergencial de saúde. Método: este estudo apresenta a combinação de uma revisão de literatura com o desenvolvimento de um modelo conceitual teórico, com intuito de preencher lacunas e propor um conteúdo para novos estudos. Originalidade: a literatura que aborda tal temática caracteriza o entendimento das percepções dos clientes como extremamente necessário, porém em comparação a percepção de qualidade do serviço, difícil de ser mensurada. Contribuições: através do modelo conceitual teórico desenvolvido é possível identificar como o serviço é avaliado em relação ao atendimento e os resultados obtidos após os tratamentos, incluindo as experiências ou expectativas dos pacientes.
\end{abstract}

Palavras-chave: Indicadores de qualidade. satisfação do cliente. atendimento emergencial.

\begin{abstract}
Hospital emergencies are places of service provision of great social importance, having high complexity and peculiarity, therefore the practice of quality acquires a specific focus and differential. The present work aims to propose the development of a theoretical model of HEALTHQUAL applicable to the context of hospital emergency services, aiming to measure the indicators of perceived quality during emergency health care. Method: this study presents the combination of a literature review with the development of a theoretical conceptual model, in order to fill in the gaps and propose content for further studies. Originality: the literature that addresses this theme characterizes the understanding of the customers' perceptions as extremely necessary, but that, in comparison to the perception of quality of service, is difficult to be measured. Contributions: through the developed theoretical conceptual model it is possible to identify how the service is evaluated in relation to the service and the results obtained after the treatments, including the experiences or expectations of the patients.
\end{abstract}

Keywords: Indicators quality. customer satisfaction. emergency hospital.

\footnotetext{
${ }^{1}$ Biomedica* - UFCSPA. E-mail: fernanda.carpeggiani@hotmail.com

${ }^{2}$ Médica*_UFCSPA. E-mail: anapaulagomes82@gmail.com

${ }^{3}$ Farmacêutica-bioquímica - UFCSPA. E-mail: ambarancelli@gmail.com

${ }^{4}$ Relações públicas* - UFCSPA. E-mail: luizaonzi@gmail.com

${ }^{5}$ Administrador* - UFCSPA. E-mail: marcelo.ufcspa@gmail.com

${ }^{6}$ Doutora em Administração pela Universidade Federal do Rio Grande do Sul - UFCSPA. Professora Adjunta A da Fundação Universidade Federal de Ciências da Saúde de Porto Alegre. E-mail: mellinaterres@gmail.com

*Atualmente cursa mestrado no Programa de Pós-graduação em Tecnologias da Informação e Gestão em Saúde -

Universidade Federal de Ciências da Saúde de Porto Alegre - UFCSPA
}

Artigo recebido em: 29 de março de 2021. Artigo aceito em 24 de novembro de 2021. 


\section{Introdução}

Para o setor de prestação de serviços, a assimilação das percepções dos clientes é primordial para que haja a convergência entre o prestador de serviço e o consumidor. Contudo, a aferição desta percepção quanto à qualidade dos serviços é difícil de ser mensurada (PELISSARI et al., 2012). Portanto, a falta de um instrumento adequado para medir as impressões e expectativas dos clientes torna ainda mais custoso a realização, pela organização, de um diagnóstico avaliativo sobre o perfil de satisfação de seus consumidores.

A satisfação pode ser entendida como o "sentimento de prazer ou de desapontamento resultante da comparação do desempenho esperado pelo produto (ou resultado) em relação às expectativas da pessoa" (MOURA; LUCE, 2004). E, também, é um indicador importante para avaliar a qualidade dos serviços em saúde, visto que oferece informações referentes a diferentes fatores do processo de atendimento estrutura, relação interpessoal, etc (ANDRADE, 2020).

O crescimento da procura por serviços de saúde impulsionada principalmente pelo envelhecimento da população torna imprescindível manter a qualidade deste produto (FANG et al., 2020). Investir na satisfação do paciente pode contribuir para a melhoria da qualidade dos serviços, bem como para bons desfechos de saúde na medida em que promove a qualidade de vida (SAMARTZIS; TALIAS, 2019).

A qualidade em saúde é orientada por uma busca de promover a gestão institucional, seja pública ou privada, de forma que se estabeleçam as definições dos processos, das melhorias, das ações corretivas e preventivas, dos indicadores de desempenho e dos treinamentos e capacitações. O foco destas atividades é a satisfação do cliente, visando que as instituições mais bem preparadas e com processos mais estruturados irão assegurar um atendimento com melhor assistência e resultado (BONATO, 2011).

Conforme a ONA (2019), há muitos anos, como por exemplo em 1902 com a $1^{\text {a }}$ guerra mundial e seus efeitos colaterais, já se falava em implementar a gestão da qualidade. Este tema é antigo e muito conhecido devido à importância e impacto que acomete as instituições, empresas, indústrias, etc (GARVIN, 1993; FERNANDES, 2011). Visto que a gestão da qualidade promove integração, comprometimento, melhoria de processos, diminuição de desperdícios, entre tantas outras vantagens, é 
importante considerar o desafio dos gestores em provocar uma mudança de cultura desde a alta administração até os profissionais que atuam diretamente nos processos (WOOD JR; URDAN, 1994).

Dentre as formas de se aplicar a gestão da qualidade em saúde, os indicadores de desempenho são a melhor forma de mensurar o desenvolvimento e resultado dos processos e melhorias, que permitem trazer base para as tomadas de decisão (ALBUQUERQUE; MARTINS, 2017). Os indicadores de desempenho são medidas com dados quantitativos, que permitem a avaliação dos processos da empresa, como resultados, políticas, impactos de ações e outros, servindo de referência para intervenções na prática (CONTROLLAB, 2008).

A capacidade dos serviços de saúde de reconhecerem suas características por meio de indicadores pode auxiliar na aquisição de desempenhos superiores, na criação de conhecimentos e na geração de evidências para orientar ações estratégicas. Segundo Prioste e Melleiro (2010), as ferramentas de gestão e de qualidade são utilizadas com a finalidade de definir, mensurar, analisar e propor soluções para os problemas que interferem no bom desempenho dos processos de trabalho.

A medição de desempenho é crucial para alcançar o sucesso do negócio. Segundo Cosenz (2013), o que não pode ser medido não pode ser controlado. Portanto, estratégias e práticas de avaliação focadas em medições de desempenho precisam ser implementadas. Além disso, tal sucesso também está relacionado ao cumprimento dos objetivos estratégicos organizacionais. Portanto, é necessária uma determinação adequada de indicadores de desempenho relevantes e suas relações com os objetivos organizacionais.

Entre as inúmeras ferramentas de gestão, os indicadores devem servir para melhorar a decisão dos gestores. Define-se como indicador, uma unidade de medida de uma atividade, com a qual se está relacionada ou ainda, uma medida final quantitativa que pode ser usada como um guia para monitorar e avaliar a qualidade de importantes cuidados providos ao paciente e as atividades dos serviços de suportes (PRIOSTE; MELLEIRO, 2010). Os Indicadores de Desempenho de Processos são também chamados de KPI (Key Performance Indicator). Trata-se de métricas que avaliam os resultados de uma organização ou de atividades específicas. A avaliação do desempenho identifica lacunas entre os resultados atuais e desejados e fornece uma indicação do 
progresso no sentido de fechar as lacunas. KPIs são comumente usados para medir e melhorar o desempenho dos sistemas de saúde (SPACKMAN et al., 2019).

Indicadores tais como de acessibilidade e de aceitabilidade estão relacionados com a atração do produto para a população. Por outro lado, indicadores de rentabilidade são importantes para viabilizar um serviço. Logo, a satisfação do usuário e a funcionalidade de um serviço afetam de forma direta ou indireta a qualidade de vida, a morbimortalidade e a sobrevida dos pacientes. A medição e o monitoramento sistemático dos indicadores de qualidade são a base para a manutenção de um serviço de saúde fundamentado em evidências (SAMARTZIS; TALIAS, 2019).

Assimilando esta carência, Parasuraman, Zeithaml e Berry (1988) descreveram uma escala, denominada SERVQUAL, focada na avaliação das percepções dos clientes em relação à qualidade dos serviços oferecidos pelas organizações. Os autores explicam que ela é composta por duas seções divididas em cinco dimensões. As seções são referentes às expectativas e percepções dos clientes, enquanto as cinco dimensões são alusivas à tangibilidade, confiabilidade, responsividade, garantia e empatia.

Ainda que a escala SERVQUAL tenha sido aplicada (com ou sem adaptações) em organizações da área da saúde (IBRAHIM, 2020; ZAREI et al., 2012), há autores que defendem a ideia de desenvolver uma escala específica para mensurar a qualidade do serviço de saúde, ou seja, HEALTHQUAL (LEE, 2017; MIRANDA et al., 2012). Neste contexto, avaliando o que há na literatura sobre SERVQUAL e HEALTHQUAL, esta pesquisa, através de um ensaio teórico, objetiva: (a) refletir sobre como as dimensões do modelo HEALTHQUAL seriam aplicadas em serviços de emergência hospitalar; e (b) propor melhorias para que a escala HEALTHQUAL possa ser utilizada em serviços de emergência hospitalar.

\section{Metodologia}

Os procedimentos metodológicos adotados nesta pesquisa buscam (1) revisar artigos relacionados às escalas SERVQUAL e HEALTHQUAL no contexto de atendimento hospitalar emergencial; (2) verificar a viabilidade e propor uma abordagem sobre os passos para o desenvolvimento de um modelo teórico de HEALTHQUAL, enquadrando-o na conjuntura emergencial de um hospital.

Acerca do teor desenvolvido neste ensaio teórico, realizou-se um levantamento de produção científica nas bases de dados Biblioteca Virtual em Saúde (BVS); Web of Science; PubMed e Science Direct, para tanto foram consideradas publicações Revista Eletrônica Gestão e Serviços v.12, n. 1, pp. 3426 - 3449, Janeiro/Junho 2021.

ISSN Online: 2177-7284 e-mail: regs@metodista.br 
realizadas no período entre janeiro/2010 e janeiro/2021. Para a elaboração desta verificação foram ponderados os conceitos de: modelo SERVQUAL, modelo HEALTHQUAL, marketing, cuidados de saúde, qualidade em saúde e emergência hospitalar, pois julga-se que o resultado desta verificação tende a demonstrar o que há de pesquisas relacionadas com o tema, bem como evidenciar a necessidade de estudos focados em um modelo de HEALTHQUAL em atendimento emergencial.

\subsection{String de busca}

Considerando as questões de pesquisas, os descritores foram definidos em português e traduzidos para inglês para, posteriormente, ser construída a String de busca. O Quadro 1 apresenta a String de busca utilizada neste estudo.

Quadro 1 Descritores utilizados no String de busca

\begin{tabular}{|c|}
\hline String \\
\hline $\begin{array}{c}\text { SERVQUAL AND (management indicators) AND marketing AND (health care OR } \\
\text { healthcare) AND (healthqual OR quality of health) AND (hospital emergency) }\end{array}$ \\
\hline
\end{tabular}

\subsection{Critérios de inclusão e exclusão}

\subsubsection{Os critérios de inclusão utilizados foram:}

a) Período entre janeiro de 2010 e janeiro de 2021;

b) Relacionados ao tema proposto sobre SERVQUAL e HEALTHQUAL para satisfação do cliente em atendimento emergencial;

c) Artigos completos disponíveis no momento da consulta;

d) Artigos com acesso gratuito;

e) Artigos nos idiomas: português ou inglês.

2.2.2 Os critérios de exclusão foram:

a) Artigos fora do período definidos no critério de inclusão;

b) Que não estivessem relacionados ao tema completo;

c) Artigos indisponíveis para leitura completa no momento da consulta.

\subsection{Seleção de estudos}

Foram selecionadas 4 bases de dados para este estudo, as quais estão elencadas, juntamente com seus respectivos resultados de seleção de busca na Quadro 2: 
Quadro 2 - Relação de seleção de dados e base de dados utilizada

\begin{tabular}{|l|c|c|}
\hline Base de dados & $\begin{array}{l}\mathbf{1}^{\mathbf{a}} \text { seleção (sem } \\
\text { filtros de período) }\end{array}$ & $\begin{array}{l}\mathbf{2}^{\mathbf{a}} \text { seleção (com filtro de período, } \\
\text { repetição e área temática) }\end{array}$ \\
\hline $\begin{array}{l}\text { Biblioteca Virtual em } \\
\text { Saúde - BVS }\end{array}$ & 0 & 0 \\
\hline Web of Science & 0 & 0 \\
\hline PubMed & 18 & 7 \\
\hline Science Direct & 14 & 5 \\
\hline
\end{tabular}

Nenhuma publicação selecionada, dentre os 12 artigos, trata especificamente da qualidade percebida pelo cliente em atendimento emergencial através de um modelo HEALTHQUAL. Entretanto, conforme explicitado na seção 6 deste artigo, os resultados apresentam o que há na literatura com tema mais próximo ao proposto por este estudo e, que de alguma forma, possa contribuir para a construção de um modelo de HEALTHQUAL em atendimento emergencial de saúde.

\section{Serviços de Emergência}

O atendimento de emergência é parte integrante do sistema de saúde e pode ser considerado uma das suas portas de entrada. O objetivo desse tipo de serviço é fornecer atendimento de emergência universal a todos, independente da condição socioeconômica, para estabilizar pacientes que têm uma doença ou lesão com risco de vida e para reduzir as consequências de mortalidade, morbidade e incapacidade evitáveis (RO et al., 2017). Ainda segundo o autor, é importante fornecer evidências para o desenvolvimento de políticas e garantia de um sistema de atendimento de emergência. Avaliar de forma integral e abrangente as carências, disponibilidades e qualidade do atendimento de emergência ajudará a compreender a extensão das necessidades não atendidas e planejar estratégias para desenvolver um serviço com bom custo-efetivo.

Um servidor do sistema de saúde de emergência hospitalar pode atender patologias agudizadas no seu curso ou condições que têm sua apresentação sempre de forma emergencial, como trauma ou parada cardíaca (KOBUSINGYE et al., 2005). A fim de poder selecionar quais são os doentes que devem ser priorizados, visto que o tempo de início de atendimento é crucial em emergências, criou-se um sistema de triagem de risco ou gravidade. A triagem de pacientes teve origem com os militares nos campos de batalha em 1800, o seu conceito formal foi introduzido nos Departamentos de Emergência dos Estados Unidos, no final da década de 50. Porém, foi na década de 
90 que o sistema de triagem passou a ser mais estudado e utilizado no mundo inteiro (ZACHARIASSE et al., 2019). Os modelos de triagem têm grande variação, mas hoje em dia o modelo mais utilizado internacionalmente é o de Manchester (Manchester Triage System - MTS) - Trabalha com algoritmos e discriminadores chaves, associados a tempos de espera simbolizados por cores. A triagem que gera ao paciente uma espera maior aumenta a sua ansiedade e sentimento de desvalia ou opressão, podendo causar insatisfação com o atendimento. O paciente com medo ou com dor costuma não lidar bem nessas situações. Uma forma de melhorar esse fluxo é ter soluções rápidas para queixas ambulatoriais ou de solução para dor, além da comunicação clara sobre os motivos para essa espera. (SMITH et al., 2009)

Em um serviço hospitalar de emergência, o paciente deve ter suas demandas clínicas identificadas de imediato, baseado na sua condição de saúde na chegada e nas doenças pré-existentes (OBERMEYER et al., 2015). O fluxo de atendimento será então direcionado baseado nessas informações para atingir a melhor entrega de saúde de forma particularizada, independente das expectativas de familiares ou do próprio paciente. Conseguir equilibrar essa forma de atendimento ao que é esperado pelo cliente é o ponto fundamental.

Medir qualidade em um sistema tão dinâmico e permeado por tantas variáveis é um grande desafio. Nos serviços de emergência de países subdesenvolvidos ou em desenvolvimento, encontramos características bem particulares em comparação aos países desenvolvidos (PIÑA et al., 2015). Ao contrário do que se vê em nações ricas, a procura por atendimento de emergência em países de média e baixa renda se dá em sua maioria por pacientes jovens e sem problemas de saúde prévios ou crônicos.

Quando a qualidade do atendimento prestado é precário e leva à morte, a procura por esse tipo de serviço pode não voltar a acontecer, ou ser feita no momento errado para o tratamento da doença. Pessoal qualificado e motivado, suprimentos adequados, produtos farmacêuticos, equipamentos, coordenação e gestão orientados para as necessidades dos pacientes são responsáveis pela qualidade do atendimento, impactando em melhora da sobrevida e diminuição da morbidade (KOBUSINGYE et al., 2005).

A organização dos serviços de saúde se dá pela integração de uma série de variáveis. Esses setores, quando perfeitamente interligados e com fluidez, entregam ao paciente um atendimento de maior qualidade, incluindo a adequada prestação de saúde (PIÑA et al., 2015). Em determinados tipos de serviços de saúde e para pacientes 
específicos, algumas dessas variáveis se sobrepõem às outras, o que torna o atendimento em saúde um sistema dinâmico. Piña et al. (2015) também afirma que a tomada de decisões parte em primeiro lugar da centralização da atenção no paciente, das suas condições de saúde e preferências. A dinâmica segue passando pela capacidade da equipe de saúde e suas decisões, em conformidade com a disponibilidade de serviços de cada prestador. Esse fluxo garante entrega de qualidade em saúde, segurança e assertividade no atendimento.

A compreensão das condições que levam o paciente a buscar a emergência hospitalar, suas expectativas e suas condições de saúde prévias e na chegada ao serviço é fundamental para direcionar recursos financeiros e humanos para a melhoria do serviço. A definição de prioridades clínicas e políticas específicas de atendimento permanecem difíceis de se estabelecer devido à escassez de dados (OBERMEYER et al., 2015). Escalas para medir a experiência de pacientes e expectativas da equipe de atendimento podem ajudar a levantar informações sobre os fluxos do serviço e as barreiras para o alcance da qualidade.

A relação médico-paciente é um fator fundamental na prestação de cuidados de saúde (HARBISHETTAR et al., 2019). Há séculos era considerada apenas uma relação entre o curador e o doente, hoje em dia passou a ser considerada como a interação entre um prestador de cuidados e o usuário do serviço. Segundo o mesmo autor, podemos considerar três modelos de dinâmica dessa relação: modelo ativo-passivo, orientaçãocooperação e participação mútua. Em sistemas assistenciais de emergência hospitalar, devido à natureza das patologias alvo desse tipo de serviço, o modelo mais utilizado seria o ativo-passivo, que consiste em curar um ser inconsciente ou inanimado, o paciente apenas recebe o tratamento sem ser questionado ou chamado a participar das decisões sobre sua saúde. Geralmente nesses setores de prestação de saúde o paciente não está consciente ou não tem condições físicas ou psicológicas para que sejam utilizados os demais modelos. As decisões terapêuticas precisam ser tomadas com rapidez e assertividade para serem efetivas (WORKU; LOHA, 2017).

Sabe-se que uma boa relação médico paciente aumenta a confiança no médico e os principais componentes da qualidade dessa relação são conhecimento, confiança, lealdade e respeito. Essa interação é construída de forma gradual e por consentimento do paciente. Em atendimento de emergência hospitalar geralmente não há tempo para a formação de vínculo e tão pouco ele ocorre por escolha do paciente, já que a maioria 
procura esse serviço de forma forçada por algum desconforto repentino em sua saúde, muitas vezes não podendo selecionar o hospital e muito menos o médico que irá lhe atender (HARBISHETTAR et al., 2019). Alguns fatores podem influenciar as bases da relação médico-paciente na emergência como, por exemplo, os médicos mais velhos são considerados mais experientes e mais confiáveis. A configuração das cadeiras no consultório também tem o papel de proporcionar a sensação de confiança e acolhimento à medida que favorecem o contato visual e a escuta das queixas de saúde.

A satisfação do paciente é percebida sobre o atendimento recebido em comparação com o cuidado esperado. Em nível hospitalar, a qualidade é desafiada pela natureza de urgência do atendimento, portanto usar a satisfação do paciente como um indicador de qualidade requer monitorar os aspectos não técnicos da qualidade desse tipo de atendimento (WORKU; LOHA, 2017). Pacientes costumam descrever o ambiente de atendimento da emergência hospitalar como um local barulhento, opressor e bastante ansiogênico. O longo tempo de espera que pode ocorrer para esse tipo de serviço de saúde também pode contribuir para a piora dessa sensação no paciente.

Segundo Smith et al. (2009), o acolhimento nesse tipo de ambiente é descrito como um fator primordial para o desenvolvimento de experiências positivas nos serviços de emergência. Muitos usuários desses serviços descrevem que se sentiram satisfeitos com o atendimento quando foram compreendidos, quando houve comunicação e empatia por parte de profissionais próprios para esse tipo de atendimento como assistentes sociais, por exemplo, ou enfermeiros. Isso facilitou a construção do vínculo com o médico e possibilitou melhores desfechos de tratamento à medida que o paciente aderiu às condutas de saúde. Além disso, a clara comunicação sobre causas e consequências dos sintomas referidos, bem como a forma de lidar com eles foi descrita como fator primordial para a sensação de acolhimento (SMITH et al., 2009).

\section{Qualidade em Serviços de Saúde}

Nos últimos anos, o setor de serviços pode perceber algumas mudanças em relação à forma de lidar com os seus clientes (AARIKA-STENROOS; JAAKKOLA, 2012). Os consumidores passaram a exigir um nível maior de qualidade em relação à prestação de serviços, o que faz com que as organizações tenham que reformular continuamente suas operações de serviços. Também, de acordo com Amaral (2011), estamos vivendo um momento em que o acesso à informação se tornou de maior 
alcance para todos os públicos, devido ao avanço das tecnologias. Com essa vantagem, ficou mais viável a utilização da informação em benefício da sociedade, unindo-a ao marketing e promovendo sistemas equilibrados.

\subsection{Satisfação do Paciente}

De acordo com Prentice (2014), a qualidade está ligada a satisfação do consumidor, pois a primeira passou a ser definida como o atender e/ou exceder as expectativas dos clientes. Portanto, para que a empresa ofereça um serviço de qualidade, ela deve satisfazer ou superar o que o cliente espera receber.

Também é possível citar como fator de desafio para os prestadores de serviço a intangibilidade, que é uma característica muito expressiva em relação à prestação de serviços, e isso significa que os clientes não podem recorrer aos seus sentidos básicos para avaliar a qualidade e os benefícios do serviço (LOVELOCK; WRIGHT, 2001). Para Davis et al. (2001), a qualidade de um serviço somente existe se o cliente confirmar a existência, e esse nível de exigência requer que, através do marketing, a organização promova exatamente o que o cliente deseja.

\subsection{Qualidade e Indicadores}

O conceito de qualidade está presente há milhares de anos na sociedade, porém recentemente está formalmente conceituado e aplicado como forma de gerenciamento, além de estar em constante evolução (GARVIN, 1993). Segundo Oliveira (2020), a obtenção de qualidade nos produtos, serviços e processos deixou de ser um diferencial competitivo e transformou-se num critério indispensável para a sobrevivência organizacional.

Conforme ABNT (2015), um sistema de gestão da qualidade auxilia a avaliação do contexto geral de uma instituição para que se possa definir o impacto da sua atividade e como ela é esperada, assim permitindo a definição dos objetivos da qualidade de uma forma clara, ao colocar o cliente em primeiro lugar, atendendo aos requisitos legais e regulamentações e os utilizando de forma eficiente. Ainda de acordo com ABNT (2015), a gestão da qualidade se baseia em sete pilares sólidos: foco no cliente, liderança, engajamento de pessoas, abordagem no processo, melhoria contínua, decisão baseada em evidências e gestão de relacionamento. Estes princípios ao serem seguidos garantem que a instituição está apta a gerar valor a seus clientes de forma consistente. 
Donabedian (2005) propôs que para uma medição adequada da qualidade de um serviço é importante distinguir entre avaliação de estrutura, processo e resultado. As avaliações de estrutura retratam o ambiente em que os cuidados de saúde ocorrem. As avaliações de processo analisam o próprio processo de prestação do cuidado e podem referenciar à prática adequada do cuidado à saúde. Já as avaliações de resultados medem os resultados do atendimento, mas sua utilidade se torna restrita devido a dificuldade de diferenciar entre a qualidade do atendimento e o resultado. Assim, a qualidade do atendimento é beneficiada de forma mais efetiva quando se trata de componentes de processo.

O monitoramento da qualidade geral do atendimento em saúde se concentrou principalmente na parte estrutural, analisando a disponibilidade de estruturas e deixando a adequação dessas subexploradas (LESLIE; SUN; KRUK, 2017).

As ferramentas que avaliam a qualidade e visam orientar as medidas de melhoria, podem ser mais efetivas se focam em processos e indicadores-chave estruturais, que avaliam se as estruturas são de qualidade suficiente para atender as necessidades dos clientes (TINMOUTH, 2017). No entanto, é imprescindível analisar além de uma única área do serviço para avaliar a qualidade dos cuidados de saúde de forma mais geral e harmonizada.

\section{SERVQUAL E HEALTHQUAL}

Existem diferenças na medição da qualidade de serviços e produtos físicos, a mensuração da primeira é mais complexa, uma vez que, ainda durante o processo de prestação ou entrega do serviço a sua qualidade e a satisfação do cliente são avaliadas (ALI; BASU; WARE, 2018).

O modelo SERVQUAL é amplamente utilizado como instrumento de mensuração de expectativa e percepção do cliente, mas enfrenta desafios na avaliação da qualidade dos serviços de saúde pelo fato de não ser específico para a área. (NEMATI et al., 2020). Neste contexto, o instrumento HEALTHQUAL se difere por considerar conceitos como, acessibilidade, cuidados, disponibilidade e resultados médicos, logo, foi introduzido como substituto para esta ferramenta na avaliação ao ter objetivo de mensurar a qualidade de serviços de saúde. 


\subsection{SERVQUAL e suas dimensões}

O modelo de escala SERVQUAL, por meio de um questionário fundamentado em cinco dimensões (tangibilidade, confiabilidade, responsividade, garantia e empatia) avalia e compara a qualidade do serviço a partir da discrepância ou lacuna entre as percepções e expectativas dos consumidores (PARASURAMAN; ZEITHAML; BERRY, 1988). Ou melhor, "[...] é um instrumento de mensuração de qualidade em serviços através da avaliação da comparação entre o que é esperado do serviço em relação ao que foi percebido do serviço." (VIEIRA; AMARAL, 2016, p. 54).

As cinco dimensões que compõem a escala SERVQUAL podem ser assim compreendidas:

A tangibilidade está associada aos atributos físicos do local, ou seja, materiais que são possíveis de serem assimilados pelos cinco sentidos do corpo humano e, também, equipamentos e pessoal (PENA et al., 2013). Sob este ponto de vista, Ali, Basu e Ware (2018) afirmam que quando um paciente chega a um ambiente hospitalar em estado de angústia, a aparelhagem, o material de comunicação e o aspecto físico das instalações são importantes para o mesmo.

A confiabilidade está relacionada à habilidade do fornecedor do serviço de operacionalizá-lo de maneira segura e precisa (PARASURAMAN; ZEITHAML; BERRY, 1988).

Já a responsividade diz respeito à prestação do serviço de maneira rápida, precisa e solícita, ou seja, está correlacionada à intenção dos colaboradores em ajudar os clientes (PENA et al., 2013).

A quarta dimensão é a garantia, a qual está associada aos funcionários e sua capacidade de gerar segurança e confiança, assim como transmitir conhecimento e cortesia (PARASURAMAN; ZEITHAML; BERRY, 1988).

Por último tem-se a empatia, que na acepção de Ali, Basu e Ware (2018) corresponde à dedicação e à atenção individualizada oferecida aos clientes pelas organizações.

De acordo com Parasuraman, Zeithaml e Berry (1988), o modelo SERVQUAL é aplicável a uma extensa variedade de serviços, contudo, pode haver necessidade de adequá-lo ou, até mesmo, complementá-lo para que o instrumento se adapte às especificidades de determinada organização. No entanto, Mosadeghrad (2012) argumenta que alguns atributos específicos relacionados à qualidade na área de saúde, 
tais como acessibilidade, carinho/cuidado (caring) e resultados médicos, não são atendidos pela escala SERVQUAL.

\subsection{HEALTHQUAL e suas dimensões}

Os autores discorrem que o HEALTHQUAL é capaz de mensurar quatro dimensões de qualidade: ambiente, empatia, eficiência e eficácia; considerando que dentro destas grandes áreas há diversos subitens determinadores de medidas. Complementarmente, Lee (2017) argumenta que o modelo HEALTHQUAL inclui fatores de mensuração de qualidade multidimensionais que se enquadram em serviços de saúde atuais.

Corrobora Barrios-Ipenza et al. (2020) ao afirmarem que o HEALTHQUAL é uma escala multidimensionalidade e suas dimensões ponderam a melhoria no atendimento, eficiência, qualidade, segurança e empatia e fidelização dos usuários.

Em estudo sobre satisfação do paciente em hospitais peruanos, Barrios-Ipenza et al. (2020) desenvolveram uma escala HEALTHQUAL composta pelos seguintes indicadores: trabalhadores da saúde; trabalhadores não relacionados à saúde; instalações, equipamentos e itens tangíveis; eficiência, por último lealdade.

Já o modelo proposto por Lee (2017) é composto por cinco componentes: empatia, tangíveis, segurança, eficiência e grau de melhorias no serviço. Enquanto o modelo de Miranda et al. (2012) considera quatro dimensões, as quais são: atributos de qualidade da equipe de saúde; medidas de eficiência; atributos de qualidade da equipe não relacionada à saúde e, também, instalações.

Como uma melhor definição da usabilidade do HEALTHQUAL, "o HEALTHQUAL é avaliado pelos pacientes em relação aos serviços de atendimento e resultados após os tratamentos, incluindo as experiências ou expectativas dos pacientes. Portanto, como o HEALTHQUAL pode ser alterado de acordo com os processos dos serviços de cuidado e os resultados do tratamento recebido, eles devem ser medidos com mais clareza para melhorar a satisfação do paciente" (LEE, 2017, p.504).

Desta forma, considerando as características peculiares do serviço de atendimento emergencial, bem como os atributos das escalas SERVQUAL e HEALTHQUAL, propõe-se as seguintes dimensões para um futuro modelo de mensuração para atendimento emergencial hospitalar: 
$\underline{\text { Responsividade, }}$ corresponde à necessidade inadiável da prestação do serviço de atendimento emergencial, ou seja, capacidade de atendimento com precisão e celeridade de resposta. Tangibilidade, compreende o aspecto tangível do ambiente e estão relacionados aos materiais, equipamentos e instalações, inclusive os materiais perceptíveis pelos cinco sentidos humanos (visão, audição, paladar, olfato e tato).

Segurança, está associada à qualificação da equipe, ao ambiente seguro para o paciente e funcionários do atendimento emergencial e, também, à confiança na prestação dos serviços. Empatia, corresponde a atenção individualizada que é dispensada aos pacientes durante a permanência deste no atendimento emergencial. Eficiência, dimensão ligada às atividades operacionais, o que inclui apoio organizacional, redução de desperdícios e tempo de espera (LEE, 2017). Considerando o contexto emergencial, está associada à facilidade do trâmite e procedimentos do hospital e, também, ao tempo de espera para o atendimento, por exemplo.

Às dimensões acima, ora apresentadas, propõe-se o acréscimo de uma inédita, a qual decidiu-se denominar de acolhimento. Esta nova dimensão está diretamente associada à qualidade da relação médico-paciente, discutida na seção 3 , deste artigo. A dimensão recém proposta não está prevista em nenhum dos instrumentos de medição de qualidade seja SERVQUAL ou HEALTHQUAL, caracterizando, assim, sua originalidade.

A dimensão de acolhimento está estritamente relacionada à confiança, à lealdade e ao respeito que o relacionamento médico-paciente proporciona aos envolvidos. Desta forma, tais aspectos podem influenciar de maneira significativa a qualidade percebida pelo paciente ao longo de sua experiência no atendimento hospitalar emergencial, bem como pode influenciar no resultado do tratamento. Quanto ao exposto, corrobora Nassar (2005, p. 9), pois na opinião desta autora “[...] pode-se considerar que a qualidade do relacionamento médico-paciente não é apenas essencial para a definição da qualidade do serviço, mas a transcende enquanto interfere na própria relação terapêutica."

As dimensões acima resumidas no Quadro 3, a seguir:

Quadro 3 - Resumo das dimensões do HEALTHQUAL-Atendimento Emergencial.

\begin{tabular}{|c|l|c|}
\hline Dimensão & $\begin{array}{l}\text { Elementos que compõem a dimensão e } \\
\text { são parâmetros para a mensuração da } \\
\text { qualidade }\end{array}$ & $\begin{array}{c}\text { Instrumento de } \\
\text { origem da dimensão }\end{array}$ \\
\hline Responsividade & Capacidade de atendimento com precisão e & SERVQUAL \\
\hline
\end{tabular}

Revista Eletrônica Gestão e Serviços v.12, n. 1, pp. 3426 - 3449, Janeiro/Junho 2021. ISSN Online: 2177-7284 e-mail: regs@metodista.br 


\begin{tabular}{|c|l|c|}
\hline & celeridade de resposta & \\
\hline Tangibilidade & Materiais; equipamentos e instalações & SERVQUAL \\
\hline Segurança & $\begin{array}{l}\text { Qualificação da equipe; ambiente seguro } \\
\text { para o paciente e funcionários; confiança na } \\
\text { prestação dos serviços }\end{array}$ & $\begin{array}{c}\text { SERVQUAL } \\
\text { (Garantia) }\end{array}$ \\
\hline Empatia & Nível da atenção individualizada & SERVQUAL \\
\hline Eficiência & $\begin{array}{l}\text { Eficiência operacional visando a prestação } \\
\text { de serviços eficazes }\end{array}$ & HEALTHQUAL \\
\hline Acolhimento & $\begin{array}{l}\text { Relacionamento } \\
\text { (confiança, lealdade e respeito) }\end{array}$ & Autores do artigo \\
\hline
\end{tabular}

Fonte: Elaborado pelos autores (2021)

Assim, as dimensões propostas para um futuro modelo conceitual/teórico são seis, a saber: responsividade; tangibilidade; segurança; empatia; eficiência e acolhimento, sugere-se como nome HEALTHQUAL-Atendimento Emergencial ou HEALTHQUAL-AE.

O objetivo do HEALTHQUAL-AE é servir de balizador para o acompanhamento da qualidade dos serviços prestados no âmbito emergencial, fornecendo aos gestores informações e indicadores de qualidade que, por sua vez, possibilitando a identificação dos pontos fracos e fortes do atendimento emergencial em saúde.

\section{Discussão}

A pesquisa foi realizada nas bases de dados BVS, Web of Science, PubMed e Science Direct, tendo em vista a conjuntura proposta neste estudo. Assim, este ensaio teórico apresenta conforme fundamentação teórica já apresentada.

Prentice e Nguyen (2020) discorrem que a abordagem sobre engajamento do cliente é muito recente na literatura, e geralmente a reflexão acontece em conexão ao marketing. E, de encontro à contextualização de Magaz-González et al., (2020), essa experiência positiva do cliente com o estabelecimento tornou-se uma estratégia chave para alcançar a vantagem competitiva de serviços.

Com intuito de observar a qualidade em relação ao serviço médico, Król e Zdonek (2021) observam que a experiência médica é, para a maioria das pessoas, relativamente limitada. Tal fato pode ocorrer devido a alguns fatores como a baixa 
disponibilidade de informações, complexidade de compreender os tratamentos, entre outros.

Para Lin, Tsai e Ho (2020), depois que um cliente passa a experimentar o primeiro estágio avaliativo em relação ao serviço ou consumo utilizado e encontra uma lacuna percebida, se as percepções destes indivíduos forem muito mais baixas do que o esperado, eles estarão expostos a lacunas de vulnerabilidades. Neste contexto os autores ressaltam a importância de estudar as expectativas versus as disparidades reais percebidas sobre o serviço. Para tanto, Trejos-Gil, Escobar e Sánchez (2020) trazem conceitos inerentes a todos os níveis de atenção à saúde: a análise de indicadores em saúde otimiza recursos que, com características únicas que geram valor ao paciente, aumentam a competitividade e consequentemente a qualidade dos serviços.

Tuchen, Arora e Blessing (2020) trazem um tema importante sobre marketing de serviços de saúde, ao concluir que o planejamento de sistemas flexíveis e resilientes deve focar em uma instituição como o "provedor de experiência" para todos os usuários, o que inclui clientes, funcionários, visitantes etc. A experiência do cliente e a qualidade do serviço são questões tão importantes de serem veiculadas, quanto a experiência geral de todos os usuários. As observações dos autores se fazem concordantes com um cenário de emergência hospitalar, em que deve ser preconizado não somente $\mathrm{o}$ atendimento aos pacientes, mas aos cuidados com funcionários e colaboradores, o que, consequentemente, gera uma satisfação mais abrangente ao cliente.

A pesquisa de Pourmohammadi et al. (2018) gira em torno de que indicadores de desempenho são ferramentas essenciais para fornecer informações valiosas aos gestores, para permitir identificar os pontos fracos e fortes de uma instituição de saúde, de forma que norteia as melhorias a serem realizadas. A seleção de indicadores mais apropriados para a gestão de indicadores hospitalares é um ponto chave para garantir um sistema mais abrangente de avaliação de desempenho. Para os autores, os grandes temas de eficiência, eficácia, produtividade e financeiro abrem margem para vários outros subtemas de indicadores, como de recursos-humanos, leitos hospitalares, produtividade do centro cirúrgico, pronto-socorro, número de pacientes hospitalizados e ambulatoriais, custos, investimentos, equidade, qualidade, responsabilidade, entre outros.

Mosadeghrad (2012) faz uma observação importante ao discorrer que a qualidade da assistência em saúde é complexa e multidimensional ao âmbito que se 
torna bilateral, pois envolve não somente as expectativas dos clientes, mas dos provedores de saúde também; assim os serviços de saúde devem atender às expectativas de ambos. Todas as partes interessadas têm suas contribuições aos indicadores de saúde, principalmente quando se trata de emergência hospitalar. O autor utiliza grandes grupos de temas para elencar seus indicadores: empatia, eficiência, eficácia e ambiente; estes vão ao encontro do que é proposto pelo modelo HEALTHQUAL, ainda que não utilizado no estudo desenvolvido por Mosadeghrad (2012).

Lei e Jolibert (2012) levantam a discussão que os pacientes estão em uma era de informação, logo se tornaram mais bem informados e, por sua vez, são exigentes quanto à qualidade que esperam receber dos serviços de saúde, o que traz importantes percepções do cliente/paciente para a construção de um melhor atendimento a nível hospitalar. A pesquisa dos autores é elencada por modelos conceituais que relacionam a qualidade percebida, lealdade e satisfação, inclusive a serviços de saúde fornecidos por hospitais, através do instrumento SERVQUAL. Os autores afirmam utilizar a escala SERVQUAL adaptada a serviços de saúde, embora seja um instrumento generalista e não focado na área da saúde.

Izugami e Takase (2016) realizaram uma pesquisa a fim de verificar se a expectativa do cliente/paciente antes da internação hospitalar corresponde a sua experiência percebida com os serviços médicos utilizados. Apesar de não se tratar especificamente de emergência hospitalar, as internações também fazem parte de possíveis desdobramentos subsequentes dos casos clínicos de serviços emergenciais. Eles utilizaram de cinco grupos de indicadores para mensurar a qualidade percebida: procedimentos médicos, explicações de profissionais médicos, comportamento de prestadores de serviços médicos, sensações somáticas e condições físicas auto percebidas. Apesar de citar o modelo SERVQUAL no artigo, eles utilizam um modelo de questionário para pesquisa qualitativa descritiva.

A despeito de não discutir particularmente sobre emergência hospitalar, salientase o artigo escrito por Shieh, Wu e Huang (2010). Os autores conduziram um estudo em um hospital taiwanês para reconhecer sete principais critérios do total 22, a partir do ponto de vista dos pacientes ou de suas famílias. O questionário foi desenvolvido pelo Show Chwam Memorial Hospital, com base no modelo SERVQUAL. Com o resultado obtido nesta primeira etapa, posteriormente, aplicou-se o método de laboratório de 
avaliação e ensaio de tomada de decisão (DEMATEL), visando identificar os principais fatores de sucesso da qualidade do serviço hospitalar.

Os resultados demonstraram que uma equipe médica de confiança com competência profissional na área da saúde é o critério de maior importância. Ademais, equipe com boa comunicação e habilidade na resolução imediata de problemas, pode causar melhor impacto do que equipamentos médicos, ou seja, estas habilidades tonificam a imagem dos pacientes quanto à equipe médica (SHIEH; WU; HUANG, 2010).

Musa-Juroš et al. (2018), testaram e propuseram o MEDQUAL, uma escala criada para avaliar simultaneamente a qualidade dos cuidados prestados pela equipe médica e os cuidados recebidos pelos pacientes em um determinado departamento do hospital. Segundo os autores, há muitos estudos sobre a qualidade de saúde em SERVQUAL, porém, eles sentiram falta de uma escala que medisse a qualidade do cuidado em saúde, em específico os serviços prestados pela equipe médica em um departamento, ao invés dos serviços de saúde em geral.

Ainda nessa proposta, a equipe propôs que este instrumento pudesse avaliar as opiniões dos pacientes sobre alguns momentos específicos do atendimento das equipes, como o nível de confiança no médico profissional, o profissionalismo da enfermeira e a organização do local.

De forma distinta, os autores Jameel et al. (2019), discorrem sobre as diferentes formas e dimensões de estudo de qualidade de serviço, mas dialogam sobre a construção de propostas subjacentes, como a avaliação da qualidade da infraestrutura física, qualidade da interação provedor-paciente, qualidade administrativa, qualidade da assistência médica e de enfermagem juntos como qualidade de serviço de cinco dimensões.

\section{Considerações finais, limitações e sugestões para estudos futuros}

Existem muitas variáveis envolvidas no processo de satisfação do cliente que incluem questões de tratamento e acompanhamento médicos, contribuindo assim para a entrega de qualidade de vida, sobrevida e saúde ao paciente. A procura de atendimento gera estresse ao cliente antes mesmo de buscar o serviço de emergência. Esse fator por si só pode aumentar o nível de insatisfação do cliente com o serviço, mesclando o descontentamento com uma situação de impotência diante de uma doença, com o 
atendimento recebido por quem deveria prover a esperança e a saúde. Um paciente satisfeito com o serviço prestado mantém-se em contato com a instituição o que implica em um bom seguimento e controle de seu estado de saúde.

$\mathrm{O}$ acolhimento do paciente principalmente no setor de triagem pode determinar uma melhora significativa na impressão do paciente sobre seu atendimento, pois implica em otimização da comunicação e da compreensão de todos os passos seguidos para diagnóstico e tratamento dentro de um setor de emergência hospitalar. Até mesmo a espera que pode ocorrer para o atendimento pode ser amenizada apenas com a abordagem correta do paciente.

Neste sentido, o acolhimento demonstrou ser uma variável importante para a medição da qualidade nos serviços de atendimento hospitalar emergencial, motivo pelo qual foi proposta sua inclusão como dimensão no novo modelo de escala de qualidade. Ademais, a mensuração da dimensão acolhimento será verificada por meio do quesito relação médico-paciente, o qual é um fator essencial na prestação serviços relacionados aos cuidados de saúde.

A aplicação de um modelo de mensuração de qualidade em atendimento de emergência pode ajudar os serviços de saúde a analisar de forma mais eficiente os fluxos de atendimento. Portanto, acredita-se que a escala ora proposta, a HEALTHQUAL-AE, através das dimensões: responsividade; tangibilidade; segurança; empatia; eficiência e acolhimento poderão suprir esta lacuna, auxiliando os gestores e trabalhadores da área da saúde a compreender melhor as implicações de um serviço de emergência hospitalar sob a perspectiva do cliente do serviço de saúde, possibilitando atingir as expectativas de satisfação do paciente, melhorando a sua experiência.

Contudo, o presente estudo apresenta como limitações: a) Trata-se de um estudo ainda limitado ao campo teórico, havendo necessidade de avaliação empírica das bases aqui propostas; b) Há pouca quantidade de estudos que abordam a mensuração da qualidade no atendimento emergencial a partir de escalas de qualidade, como por exemplo, SERVQUAL e HEALTHQUAL, dificultando a pesquisa sobre este tema.

Como sugestões para estudos futuros, considerando que este artigo propôs as bases para o desenvolvimento de um modelo de mensuração de qualidade no contexto hospitalar de atendimento emergencial, sugere-se o desenvolvimento do questionário HEALTHQUAL-AE e sua aplicação em uma pesquisa empírica para avaliar sua aderência e viabilidade prática diante do ambiente emergencial. 


\section{REFERÊNCIAS}

AARIKA-STENROOS, L.; JAAKKOLA, E. Value co-creation in knowledge intensive busi-ness services: a dyadic perspective on the join problem solving process. Industrial Marketing Management, v.41, p. 15-26, 2012.

ASSOCIAÇÃO BRASILEIRA DE NORMAS TÉCNICAS (ABNT) (org.). ABNT NBR ISO 9001:2015: como usar. 2015. Disponível em:

http://www.abnt.org.br/publicacoes2/category/145-abnt-nbr-iso-9001. Acesso em: 29 jan. 2021.

ALBUQUERQUE, C.; MARTINS, M. Indicadores de desempenho no Sistema Único de Saúde: uma avaliação dos avanços e lacunas. Saúde em Debate, [S.L.], v. 41, n. , p. 118-137, mar. 2017. FapUNIFESP (SciELO). http://dx.doi.org/10.1590/0103$\underline{11042017 \mathrm{~s} 10 .}$.

ALI, S. S.; BASU, A.; WARE, N. Quality measurement of Indian commercial hospitals - using a SERVQUAL framework. Benchmarking: An International Journal, [s. l.], v. 25, n. 3, p. 815-837, 2018. DOI: https://doi.org/10.1108/BIJ-05-20160060. Disponível em: https://www-

emerald.ez41.periodicos.capes.gov.br/insight/content/doi/10.1108/BIJ-05-20160060/full/html. Acesso em: 25 jan 2021.

AMARAL, S. A. do. Marketing da informação: abordagem inovadora para entender o mercado e o negócio da informação. Ciência da Informação, Brasília, DF, v. 40, n. 1, p. 85-98, jan./abr., 2011.

ANDRADE, Fabíola Bof de; PINTO, Rafaela da Silveira. Fatores associados à insatisfação dos usuários dos centros de especialidades odontológicas do Brasil em 2014: estudo transversal. Epidemiol. Serv. Saúde, Brasília, v. 29, n. 3, e2019429, 2020 . Disponível em http://www.scielo.br/scielo.php?script=sci_arttext\&pid=S223796222020000300306\&lng=pt\&nrm=iso. Acesso em 10 jan. 2021. Epub 08-Jun-2020.

BARRIOS-IPENZA, F. et al. Patient satisfaction in the peruvian health services: Validation and application of the HEALTHQUAL scale. International Journal of Environmental Research and Public Health, [s. l.], v. 17, n. 14, p. 1-15. 2020.

Disponível em: https://doi.org/10.3390/ijerph17145111. Acesso em: 04 fev. 2021.

BONATO, Vera Lucia. Gestão de qualidade em saúde: melhorando a assistência ao cliente. O Mundo da Saúde, São Paulo, v. 35, n. 5, p. 319-331, 2011.

CONTROLLAB (Brasil). Boletim qualifique. ed 23: indicadores laboratoriais na prática. 2008. Disponível em:

https://controllab.com/qualifique/pop_ed23_indicadores_laboratoriais.htm. Acesso em: 08 jan. 2021.

COSENZ, F. (2013). The Entrepreneurial University: A Preliminary Analysis of the Main Managerial and Organisational Features towards the Design of Planning \& Control Systems in European Academic Institutions. Management Research and Practice, 5 (4), 19-36.

DAVIS, M; et al. Fundamentos da administração de produção. 3. ed. Porto Alegre: Bookman, 2001. 
DONABEDIAN, Avedis. Evaluating the quality of medical care. The Milbank memorial fund quarterly, v. 44, n. 3, p. 166-206, 1966.

FANG E. F. et al. "A research agenda for ageing in China in the 21st century (2nd edition): Focusing on basic and translational research, long-term care, policy and social networks." Ageing research reviews vol. 64 (2020): 101174. doi:10.1016/j.arr.2020.101174.

FERNANDES, W. A. O Movimento da Qualidade no Brasil. BRASIL/MDIC/INMETRO. Essencial Idea Publishing. 2011.

GARVIN, David A. Manufacturing strategic planning. California Management Review, v. 35, n. 4, p. 85-106, 1993.

HARBISHETTAR, Vijaykumar et al. The enigma of doctor-patient relationship. Indian journal of psychiatry, [S.L.], n. 61, Suppl 4, p. S776-S781, abr. 2019. doi: doi:10.4103/psychiatry.IndianJPsychiatry_96_19.

IBRAHIM, M. S. Validating service quality (SERVQUAL) in healthcare: measuring patient satisfaction using their perceptions in Jordan. Journal of Information \& Knowledge Management, [s. l.], v. 19, n. 01, p. 1-13, 2020. DOI: https://doi.org/10.1142/S0219649220400213. Disponível em: https://www.worldscientific.com/doi/epdf/10.1142/S0219649220400213. Acesso em: 25 jan. 2021.

IZUGAMI, Satoko; TAKASE, Kozo. Consumer Perception of Inpatient Medical Services. Plos One, [S.L.], v. 11, n. 11, 10 nov. 2016. Public Library of Science (PLoS). http://dx.doi.org/10.1371/journal.pone.0166117.

JAMEEL, Arif et al. Improving Patient behavioral Consent through Different Service Quality Dimensions: assessing the mediating role of patient satisfaction. International Journal Of Environmental Research And Public Health, [S.L.], v. 16, n. 23, 27 nov. 2019. MDPI AG. http://dx.doi.org/10.3390/ijerph16234736.

KOBUSINGYE O. C. et al. Emergency medical systems in low- and middle-income countries: recommendations for action. Bull World Health Organ. 2005 Aug;83(8):626-31. Epub 2005 Sep 22. PMID: 16184282; PMCID: PMC2626309.)

KRÓL, Karol; ZDONEK, Dariusz. The Quality of Infectious Disease Hospital Websites in Poland in Light of the COVID-19 Pandemic. International Journal of Environmental Research and Public Health, v. 18, n. 2, p. 642, 2021.

LEE, D. H. HEALTHQUAL: a multi-item scale for assessing healthcare service quality. Service Business, v. 11, n. 3, p. 491-516, 2017.

LEI, Ping; JOLIBERT, Alain. A three-model comparison of the relationship between quality, satisfaction and loyalty: an empirical study of the chinese healthcare system. Bmc Health Services Research, [S.L.], v. 12, n. 1, p. 1-11, 30 nov. 2012. Springer Science and Business Media LLC. http://dx.doi.org/10.1186/1472-6963-12-436.

LESLIE, Hannah H.; SUN, Zeye; KRUK, Margaret E. Association between infrastructure and observed quality of care in $\mathbf{4}$ healthcare services: A crosssectional study of 4,300 facilities in 8 countries. PLoS medicine, v. 14, n. 12, p. e1002464, 2017. 
LIN, Paohui; TSAI, Hsientang; HO, Tzuya. Food Safety Gaps between Consumers' Expectations and Perceptions: Development and Verification of a Gap-Assessment Tool. International Journal of Environmental Research and Public Health, v. 17, n. 17, p. $6328,2020$.

LOVELOCK, C.; WRIGHT, L. Serviços: marketing e gestão. São Paulo: Saraiva, 2001.

MAGAZ-GONZÁLEZ, Ana $\mathrm{M}^{\mathrm{a}}$ et al. Overall Quality of Sporting Events and Emotions as Predictors of Future Intentions of Duathlon Participants. Frontiers in Psychology, v. 11, 2020.

MIRANDA, F. J. et al. Primary health care services quality in Spain: A formative measurement approach using PLS path modeling. International Journal of Quality and Service Sciences, [s. l.], v. 4, n. 4, p. 387-398, 2012. DOI: https://doi.org/10.1108/17566691211288368. Acesso em: 06 fev. 2021.

MOSADEGHRAD, A. M. A conceptual framework for quality of care. Materia socio-medica, [s. l.], v. 24, n. 4, p. 251-261, 2012. DOI:

https://doi.org/10.5455/msm.2012.24.251-261. Acesso em: 06 fev. 2021.

MUSA-JUROŁ, Ksenija et al. Measuring Healthcare Quality - Paradigm of MEDQUAL. Acta Clinica Croatica, [S.L.], v. 57, n. 2, p. 235-242, jun. 2018. Sestre Milosrdnice University Hospital Center (KBC Sestre milosrdnice). http://dx.doi.org/10.20471/acc.2018.57.02.02

MOURA, Gisela Maria Schebella Souto de; LUCE, Fernando Bins. Encontros de serviço e satisfação de clientes em hospitais. Rev. bras. enferm., Brasília, v. 57, n. 4, p. 434-440, ago. 2004. Disponível em http://www.scielo.br/scielo.php?script=sci arttext\&pid=S003471672004000400010\&lng=pt\&nrm=iso. Acesso em 10 jan. 2021.

NASSAR, M. R. F. Comunicação e Humanização: a reconstrução do relacionamento médico - paciente como critério de qualidade na prestação de serviço. Contemporânea (Título não-corrente), [s.1.], v. 3, n. 2, p. 1-13, nov. 2015. DOI:

https://doi.org/10.12957/contemporanea.2005.17138. Disponível em: https://www.epublicacoes.uerj.br/index.php/contemporanea/article/view/17138. Acesso em: 21 mar. 2021.

NEMATI, R. et al. Hospital service quality based on HEALTHQUAL model and trusting nurses at Iranian university and non-university hospitals: a comparative study. BMC nursing, v. 19, n. 1, p. 1-9, 2020.

OBERMEYER Z, et al. Emergency care in 59 low- and middle-income countries: a systematic review. Bull World Health Organ. 2015;93(8):577-586G.

doi:10.2471/BLT.14.148338.

OLIVEIRA, Otávio J. Curso básico de gestão da qualidade. Cengage Learning, 2020.

ONA. ORGANIZAÇÃO NACIONAL DE ACREDITAÇÃO (Brasil). Gestão da

Qualidade em Saúde: sua história e grandes mudanças. Sua História e Grandes

Mudanças. 2019. Disponível em: https://www.ona.org.br/uploads/e-Book_-

_Series_20_anos_-_01.pdf. Acesso em: 08 jan. 2021.

PARASURAMAN, A.; ZEITHAML, V. A.; BERRY, L. L. SERVQUAL: a multipleitem scale for measuring consumer perceptions of service quality. Journal of Retailing, [s. l.], v. 64, n. 1, 1988.

Revista Eletrônica Gestão e Serviços v.12, n. 1, pp. 3426 - 3449, Janeiro/Junho 2021.

ISSN Online: 2177-7284 e-mail: regs@metodista.br 
PELISSARI, A. S. et al. Aplicação e avaliação do modelo Servqual para analisar a qualidade do serviço. InterSciencePlace, [s. l.], ed. 23, v. 1, p. 1 - 24, out./dez. 2012. DOI: http://dx.doi.org/10.6020/1679-9844/2301. Disponível em:

http://www.interscienceplace.org/isp/index.php/isp/article/view/219/216. Acesso em: 7 jan. 2021.

PENA, M. M. et al. O emprego do modelo de qualidade de Parasuraman, Zeithaml e Berry em serviços de saúde. Revista da Escola de Enfermagem da USP, [s. l.], v. 47, , n. 5, p. 1235 - 1240, 2013. DOI: https://doi.org/10.1590/S0080-623420130000500030. Disponível em: https://www.scielo.br/pdf/reeusp/v47n5/pt_0080-6234-reeusp-47-051227.pdf. Acesso em: 07 jan. 2021.

PIÑA I. L. et al. A framework for describing health care delivery organizations and systems. Am J Public Health. 2015 Apr;105(4):670-9. doi:

10.2105/AJPH.2014.301926. Epub 2014 Jun 12. PMID: 24922130; PMCID:

PMC4358211.

POURMOHAMMADI, Kimia et al. A comprehensive map of the evidence on the performance evaluation indicators of public hospitals: a scoping study and best fit framework synthesis. Cost Effectiveness and Resource Allocation, v. 16, n. 1, p. 1-22, 2018.

PRENTICE, Catherine. Who stays, who walks, and why in high-intensity service contexts. Journal of Business Research, v. 67, n. 4, p. 608-614, 2014.

PRENTICE, Catherine; NGUYEN, Mai. Engaging and retaining customers with AI and employee service. Journal of Retailing and Consumer Services, v. 56, p. 102186, 2020 .

PRIOSTE, Poliana Pertence e MELLEIRO Marta Maria. (2010) Implantação de ferramenta de gestão de qualidade em Hospital Universitário. Revista Escola de Enfermagem USP: 44(4).

RO Y. S. et al. Evaluation of demands, usage and unmet needs for emergency care in Yaoundé, Cameroon: a cross sectional study. BMJ Open 2017;7:e014573. doi:10.1136/bmjopen-2016-014573.

SAMARTZIS, L., TALIAS, M. A. Assessing and Improving the Quality in Mental Health Services. Int J Environ Res Public Health. 2019;17(1):249. Published 2019 Dec 30. doi:10.3390/ijerph17010249.

SHIEH, J. I.; WU, H. H.; HUANG, K. K. A DEMATEL method in identifying key success factors of hospital service quality. Knowledge-Based Systems, [s. l.], v. 23, n. 3, p. 277-282, 2010. Disponível em: https://doi.org/10.1016/j.knosys.2010.01.013. Acesso em: 05 fev. 2021.

SMITH A. K. et al. Emergency department experiences of acutely symptomatic patients with terminal illness and their family caregivers. J Pain Symptom Manage. 2010;39(6):972-981. doi:10.1016/j.jpainsymman.2009.10.004

SPACKMAN, E. et al. (2019) Developing key performance indicators for prescription medication systems.PLOS ONE, San Francisco, Califórnia, EUA.

TINMOUTH, Jill. Unpacking quality indicators: how much do they reflect differences in the quality of care? Bmj Quality \& Safety, [S.L.], v. 27, n. 1, p. 4-6, 26 set. 2017. BMJ. http://dx.doi.org/10.1136/bmjqs-2017-006782. 
TREJOS-GIL, Carlos Andrés; ESCOBAR, Huber Yesid Castro; SÁNCHEZ, Omar Augusto Amador. Measurement indicator to psychology online in Latin American in pandemic times. Revista Latina de Comunicación Social, n. 78, p. 457-476, 2020.

TUCHEN, Stefan; ARORA, Mohit; BLESSING, Lucienne. Airport user experience unpacked: Conceptualizing its potential in the face of COVID-19. Journal of air transport management, v. 89, p. 101919, 2020.

VIEIRA, B. K. G.; AMARAL, T. M. Aplicação da ferramenta SERVQUAL: Um caso prático no Hospital Universitário da Universidade Federal do Vale do São Francisco. Produção em Foco, [s. l.], v. 6, n. 2, p. 56-65, 2016. DOI: https://doi.org/10.14521/P2237-5163.2016.0010.0006. Disponível em: https://producaoemfoco.org/producaoemfoco/article/view/315/211. Acesso em: 25 jan. 2021.

WOOD JR., T.; URDAN, F. T. Gerenciamento da Qualidade Total: uma Revisão Crítica. RAE-Revista de Administração de Empresas, v. 34, n. 6, nov-dez, p.46-59, 1994. http://dx.doi.org/10.1590/S0034-75901994000600006

WORKU, M.; LOHA, E. Assessment of client satisfaction on emergency department services in Hawassa University Referral Hospital, Hawassa, Southern Ethiopia. BMC Emerg Med. 2017;17(1):21. Published 2017 Jun 27. doi:10.1186/s12873-017$0132-7$

ZACHARIASSE J. M, van der Hagen V, Seiger N, Mackway-Jones K, van Veen M, Moll HA. Performance of triage systems in emergency care: a systematic review and meta-analysis. BMJ Open. 2019;9(5):e026471. Published 2019 May 28. doi:10.1136/bmjopen-2018-026471

ZAREI, A. et al. Service quality of private hospitals: The Iranian Patients' perspective. BMC Health Services Research, [s. l.], v. 12, n. 1, p. 1-7, 2012. DOI: https://doi.org/10.1186/1472-6963-12-3. Disponível em: https://www.ncbi.nlm.nih.gov/pmc/articles/PMC3306759/pdf/1472-6963-12-31.pdf. Acesso em: 27 jan. 2021. 